\title{
Usabilidad de redes sociales con propósitos académicos en educación superior
}

\author{
Joel Angulo-Armenta ${ }^{\star}$, Pablo A. Sandoval- Mariscal' ${ }^{1}$, Carlos A. Torres-Gastelú ${ }^{2}$ y Ramona I. García-López ${ }^{1}$ \\ (1) Departamento de Educación, Instituto Tecnológico de Sonora, Ciudad Obregón, Sonora, México. \\ (correo-e: joangulo@potros.itson.edu.mx; imelda.garcia@potros.itson.edu.mx; \\ pablo.sandoval163068@potros.itson.edu.mx) \\ (2) Facultad de Administración, Universidad Veracruzana, Veracruz, México. (correo-e: ctorres@uv.mx)
}

* Autor a quien debe ser dirigida la correspondencia.

Recibido May. 4, 2021; Aceptado Jun. 14, 2021; Versión final Ago. 14, 2021, Publicado Dic. 2021

\begin{abstract}
Resumen
El objetivo de este estudio fue determinar el uso de plataformas de redes sociales con fines académicos por parte de estudiantes de dos universidades mexicanas. Se realizó un estudio cuantitativo descriptivo comparativo, con un diseño transversal no experimental; la muestra fue no aleatoria bajo el criterio intencional de 593 estudiantes de ambas instituciones; además, se administró una escala para medir las dimensiones de efectividad, eficiencia y satisfacción. La red social elegida fue Facebook ya que permite crear comunidades virtuales con fines educativos. Los resultados indicaron que los alumnos mostraron interés por las redes sociales para actividades de esparcimiento, pero no recomiendan su uso como plataformas de aprendizaje. El aporte de este estudio proporcionó un análisis descriptivo, evidencia empírica y estadística sobre el uso de Facebook. Se concluye que los estudiantes asocian las redes sociales con el esparcimiento y no con la educación; y, por lo tanto, prefieren separar ambas actividades.
\end{abstract}

Palabras clave: usabilidad de redes; comunidades virtuales; educación superior; redes sociales

\section{Usability of social networks for academic purposes in higher education}

\begin{abstract}
The objective of this study was to determine the use of social networking platforms for academic purposes by students from two Mexican universities. A descriptive comparative quantitative study was carried out with a non-experimental cross-sectional design; it was a non-probability sampling under the intentional criterion of 593 students from both institutions; in addition, a scale was administered to measure the dimensions of effectiveness, efficiency, and satisfaction. The social network chosen was Facebook since it allows the creation of virtual communities for educational purposes. The results indicated that students showed interest in social networks for leisure activities, but do not recommend their use as learning platforms. The contribution of this study provided a descriptive analysis, empirical and statistical evidence on the use of Facebook. It is concluded that students associate social networks with leisure and not with education; therefore, they prefer to separate both activities.
\end{abstract}

Keywords: network usability; virtual communities; higher education; social networks. 


\section{INTRODUCCIÓN}

En este apartado, se puntualizan los antecedentes de estudios empíricos relacionados con el objeto de estudio, así como el objetivo general, problema y pregunta de investigación. Los avances tecnológicos digitales de las últimas tres décadas han encaminado a los diversos actores educativos a diseñar e integrar nuevos escenarios pedagógicos en todos los niveles educativos. Las comunidades en línea aplicadas en la formación de estudiantes, son un ejemplo que puede observarse hoy en día. Estos grupos virtuales son considerados foros de comunicación interactiva basados en el uso de la Internet, donde la participación se basa en la comunicación mediada por una computadora. El éxito de los espacios como las redes sociales, foros de discusión, wikis, blogs y micro-blogs depende de que los participantes se unan y tengan una interacción permanente; esto se hace en las principales plataformas de medios sociales capaces de crear comunidades virtuales. En este sentido, la existencia de una comunidad depende de las relaciones establecidas por la interacción de los miembros y el intercambio de recursos a través de la red; dichas acciones establecen canales de comunicación intrapersonal de forma sincrónica y asincrónica que permite a los usuarios comunicarse e interactuar con una amplia gama de usuarios, en donde incontables fenómenos sociales del mundo real, se dan de forma análoga en las comunidades virtuales.

La educación es uno de los procesos educativos que más se ha relacionado con las plataformas en línea. Desde un ambiente formal, se pueden identificar plataformas públicas y privadas que son didácticas y flexibles donde el estudiante puede gestionar contenidos educativos, algunas de ellas son: Moodle, Edmodo, Blackboard, Schoology, entre otros sistemas de administración del aprendizaje (Rodríguez et al., 2018). Por su parte, en los ambientes informales, están los grupos de redes sociales como WhatsApp, correo electrónico, YouTube, Facebook, foros especializados y diversas aplicaciones móviles que sirven de esparcimiento, aunque hoy en día, estos espacios son de uso frecuente para actividades escolares entre alumnos universitarios. Esto se logra mediante el compromiso del estudiante cuando reconoce como significativo la integración de la tecnología educativa puesto que las actividades colaborativas, contenidos y tareas se encuentran relacionadas con la vida real y proporcionan a los estudiantes diversos mecanismos para una activa participación (Bond y Bedenlier, 2019). Lo antes expuesto adquiere mayor relevancia cuando el proceso de aprendizaje se da a través de dispositivos con conexión a la Internet, la diversidad de sitios de redes sociales digitales y las interacciones entre los estudiantes.

En la literatura científica existen diversos estudios relacionados con la usabilidad de las tecnologías digitales por los jóvenes, tales como Álvarez-Cruces et al. (2019) y Guillén-López (2019). Al respecto de la usabilidad de las redes sociales en el proceso de aprendizaje, Gómez-Hurtado et al., (2018), encontraron resultados que señalan que las redes sociales son un recurso didáctico que promueve y facilita la colaboración entre alumnos y docentes. En este sentido, Abbas et al., (2019) afirma que las características positivas de dichas plataformas incluyen compartir opiniones con otros; sensibilización; compartir conocimiento, construcción de relaciones, identidad, reputación y contactos; mejora de la influencia social; y el desarrollo de otras habilidades de comunicación. Las redes sociales han contribuido a mejorar el aprendizaje y las interacciones sociales, así como la compartición de información. Por su parte, Reyes-Garcés et al., (2018), en un estudio sobre usabilidad y aceptación tecnológica de una red social por alumnos, observaron que el uso del Facebook es la mejor alternativa como una plataforma para el aprendizaje. De igual forma, Llorens y Capdeferro (2011) señalan que Facebook facilita y potencializa procesos de construcción de conocimientos por medio del intercambio y uso de información, lo que ayuda a la creación de comunidades de aprendizaje.

Por otra parte, es un hecho que las redes sociales en la educación universitaria se están usando como un recurso tecno-educativo en el diseño y desarrollo del currículo; contribuyendo en el proceso de enseñanza y aprendizaje, dinamizando, innovando las clases tradicionales y las pedagogías implícitas (Manca y Ranieri, 2016a). Finalmente, hay otras indagaciones orientadas al uso de las redes sociales, comunidades virtuales e interacción digital en la formación universitaria (Chávez-Márquez y Gutiérrez-Diez, 2015; Zachos et al., 2018), las cuales coinciden en la contribución de las redes en el proceso educativo. De la misma manera, se han identificado barreras hacia el uso de redes sociales en el aula relacionadas con la infraestructura de las instituciones educativas (Tulinayo et al., 2018), con elementos culturales, como modelos pedagógicos tradicionales y falta de apoyo técnico (Manca y Ranieri, 2016b); profesores con una tendencia a usar las redes sociales con fines personales y profesionales (Manca y Ranieri, 2016b) y factores individuales como el balance entre las actividades académicas y la vida social en línea, particularmente en los estudiantes de nuevo ingreso (Junco, 2015).

En el contexto de la educación pública universitaria en México, la Organización para la Cooperación y el Desarrollo Económico (OCDE, 2019) establece que este sector tiene el $70 \%$ de los estudiantes en las 3,762 instituciones del país; además que la educación superior está creciendo y el $26 \%$ de sus estudiantes obtendrá un título universitario. Asimismo, a la fecha de este documento citado, aproximadamente 500000 egresados están laborando; empero por la situación pandémica de la COVID-19 en 2020, los egresados participan menos en el mercado del trabajo. Sin duda y asociado a lo descrito, la educación universitaria tiene hoy en día 
grandes retos para formar profesionistas competentes y visionarios en una economía moderna mundial, donde las tecnologías digitales y su interacción a través de estas, son parte sustancial en el proceso de enseñanza y aprendizaje.

Por otra parte, la Asociación de Internet MX (AIMX, 2019), en el 15ำ estudio sobre los hábitos de los usuarios de la Internet 2018 en México, establece que el $18 \%$ en edad universitaria entre 18 y 24 años de edad usan la Internet; asimismo, de los usuarios mexicanos de Internet, el $82 \%$ acceden a las redes sociales, el $78 \%$ envían y reciben mensajes instantáneos y el $51 \%$ realizan video conferencias. Estos datos muestran un panorama general sobre el uso de las redes sociales en jóvenes universitarios mexicanos.

Referente al contexto de las instituciones públicas de educación superior participantes en este estudio, el Instituto Tecnológico Superior de San Andrés Tuxtla (ITSSAT), ubicado en el Estado de Veracruz, México, pertenece al Sistema Tecnológico Nacional de México ubicado en la zona sur del Estado; cuenta con una matrícula de 2555 alumnos inscritos a nivel pregrado (licenciatura) en el periodo 2018-2019 en ocho ingenierías y una licenciatura en administración (ITSSAT, 2019). Por su parte, el Instituto Tecnológico de Sonora (ITSON), es una universidad pública estatal, ubicada en la zona sur del Estado de Sonora, México, y cuenta con una matrícula de 17,300 alumnos inscritos a nivel licenciatura en el periodo 2018-2019 (ITSON, 2019). En las dos universidades, la integración y uso de las tecnologías digitales en las actividades escolares es recurrente, por lo que es importante precisar que los alumnos universitarios realizan interacción a través de las redes sociales, sea para esparcimiento o como apoyo para las actividades escolares.

Con relación a la usabilidad, una de las perspectivas teóricas y empíricas relacionada con este término para sistemas interactivos, fueron planteadas en la norma ISO 9241 - 11. Ergonomics of human - system interaction (2018). En este documento se establece que la usabilidad está relacionada con el resultado de interactuar con un sistema, producto o servicio. En este sentido, el concepto de usabilidad que puede ser aplicable para el manejo de plataformas y aplicaciones de redes sociales o espacios colaborativos basados en la interacción de los usuarios a través de Internet. Dicho de otra manera, comúnmente se puede entender como la facilidad de uso de un programa, en términos de efectividad, eficiencia y satisfacción con el uso del mismo. Dichos atributos permiten adaptar los sistemas a los requisitos de los estudiantes (Parsazadeh et al., 2018).

Ahora bien, el constructo de usabilidad se limitó a la interactividad en comunidades virtuales en las redes sociales (Facebook) entre estudiantes de dos universidades mexicanas, analizándose las dimensiones de efectividad, eficiencia y satisfacción (Cattaneo et al., 2015; Iqbal y Warraich, 2012), a diferencia de los estudios de usabilidad basados en los registros de plataformas de redes sociales o LMS (Harrati et al., 2016). Por su parte, Garzón (2020) clasifica las comunidades virtuales en comunidades de aprendizaje basadas en tareas, en la práctica y en el conocimiento. Ambas ideas descritas, son de importancia ya que hoy en día los estudiantes son entes interactivos en las redes sociales digitales, lo que hace evidente el uso que le dan para las tareas escolares en la universidad.

Los anteriores planteamientos dieron la pauta sobre la importancia y factibilidad de realizar un estudio en dos universidades mexicanas, que ayudase a documentar un vacío en el conocimiento como es identificar y comparar el uso que los estudiantes universitarios tienen de las redes sociales para sus actividades académicas en aspectos de las dimensiones de efectividad, eficiencia y satisfacción. Por lo anterior, el objetivo de este estudio fue determinar la usabilidad de plataformas de redes sociales con fines académicos en estudiantes universitarios mexicanos. De lo anterior se derivó la siguiente pregunta de investigación ¿en qué grado los estudiantes de dos universidades mexicanas consideran que las redes sociales se usan para fines académicos?

\section{MÉTODO}

En esta sección, se describe el diseño metodológico del estudio, que consiste en: el tipo de estudio, los participantes, el procedimiento y el instrumento administrado con su validación.

\section{Tipo de estudio}

El estudio tuvo un alcance cuantitativo, fue de tipo descriptivo comparativo con un diseño transversal no experimental, la muestra fue no probabilística por conveniencia compuesta por estudiantes de pregrado de dos universidades mexicanas, una estatal en una zona urbana en un Estado al noroeste de México, y la otra federal en una zona rural en un Estado al sureste del país.

\section{Participantes}

La muestra fue no probabilística, los participantes fueron estudiantes inscritos en pregrado de dos universidades mexicanas, una estatal en una zona urbana en un Estado al noroeste de México y otra federal en una zona rural 
en un Estado al sureste del país, de los cuales 282 fueron mujeres (47.5\%) y 311 hombres (52.5\%), con edades comprendidas entre los 18 y 25 años cumplidos $(M=20.25$, $D E=1.43)$, que cursaban desde el segundo hasta el décimo semestre al momento del estudio. Los alumnos de acuerdo a su carrera pertenecían a las áreas de: Ciencias Sociales y Humanidades (17.5\%), Ciencias Económico-administrativas (54 \%), Ciencias de Ingeniería y Tecnología (20.9\%) y Recursos Naturales (7.6\%). Las características de los participantes se resumen en la Tabla 1.

\section{El análisis de datos observados}

Fue un trabajo de gabinete detallado, se hizo a través de programas computaciones como el SPSS, donde los modelos estadísticos fueron tratados con estadística descriptiva e inferencial.

Tabla 1: Descripción demográfica de los participantes.

\begin{tabular}{|l|c|c|}
\hline Atributos & $N$ & $\%$ \\
\hline ITSON & 329 & 55.48 \\
\hline Hombres & 180 & $54.7 \%$ \\
\hline Mujeres & 149 & $45.3 \%$ \\
\hline Edad & $20.09(\mathrm{DE}=1.69)$ & \\
\hline ITSSAT & 264 & 44.52 \\
\hline Hombres & 131 & $49.6 \%$ \\
\hline Mujeres & 133 & $50.4 \%$ \\
\hline Edad & $20.44(\mathrm{DE}=1.17)$ & \\
\hline
\end{tabular}

\section{Procedimiento}

Con el fin de garantizar el acceso al campo para la recolección de datos: 1) se solicitó permiso a las autoridades de las dos universidades; 2) se le explicó el propósito del estudio y la confidencialidad de los datos; 3) a los estudiantes de pregrado se les leyó el objetivo y aceptaron el consentimiento informado; 4) se les explicó de manera breve la estructura, indicaciones y el tiempo del instrumento; y 5) finalmente, se hizo el compromiso con las autoridades educativas de ambas universidades, de entregar un informe ejecutivo de los resultados. Después del acceso al campo, se realizó un análisis detallado de los datos primero para determinar la validez de constructo a través del Análisis Factorial Exploratorio y Confirmatorio y la consistencia interna mediante el Alfa de Cronbach. Finalmente se realizaron análisis estadísticos descriptivos e inferenciales para determinar las medidas de los componentes de usabilidad y la comparación entre las poblaciones mediante software estadístico SPSS 23 y AMOS 25; cabe mencionar que el tamaño del efecto y la potencia estadística fueron calculados con G*Power 3.1.9.2. Tanto el instrumento como las propiedades psicométricas del instrumento se presentan a continuación, mientras que los análisis descriptivos y la comparación de medias se presentan en el apartado de resultados.

\section{Instrumento}

Se diseñó y validó un instrumento para medir la usabilidad de plataformas de redes sociales digitales (Facebook, para fines de este estudio) para actividades académicas en estudiantes universitarios. Para la realización de este estudio, se utilizó el comportamiento recordado donde los sujetos emiten un juicio de valor basándose en su experiencia y percepciones. El diseño del cuestionario se realizó mediante un banco de preguntas que incluyen tres dimensiones del constructo de usabilidad enfocadas a la realización de actividades académicas, tales como la efectividad, eficiencia y satisfacción. La dimensión efectividad, se definió como la medida en que la plataforma de redes sociales se considera útil para cumplir las asignaciones; la eficiencia, se puntualizó como la facilidad y rapidez y la satisfacción, se especificó como la experiencia agradable al usarlas. Para las opciones de respuesta se usó una escala de Likert de 5 niveles: $1=$ Totalmente en desacuerdo, $2=$ En desacuerdo, $3=$ Neutral, $4=$ De acuerdo y $5=$ Totalmente de acuerdo. Con relación a la validez de contenido, los instrumentos fueron sometidos al jueceo de cinco expertos en el área de educación a distancia y tecnología instruccional. La validez de constructo se determinó mediante un Análisis Factorial Exploratorio (AFE) y el Análisis Factorial Confirmatorio (AFC).

\section{Análisis Factorial Exploratorio}

El determinante de la matriz de correlaciones derivado fue de .002; por su parte, la prueba de esfericidad de Bartlett resultó significativa con $\mathrm{X}^{2}(190)=2441.16 ; \mathrm{p}<.001 ; \mathrm{y}$ la medida de Kaiser-Meyer-Oklin (KMO) tuvo una buena adecuación muestral (.90) de los datos. Los resultados en una primera aproximación con un análisis factorial por el método de componentes principales con rotación Varimax y criterio de selección de las cargas factoriales mayores a .30, donde se encontró que algunos ítems se sobreponían en la matriz de componentes, eliminando los ítems efectividad 1 y 4 ; eficiencia 1 y 7 . Posteriormente, se realizó una segunda ronda de análisis, bajo los mismos criterios que la primera aproximación, excluyendo los cuatro ítems mencionados. Se encontró que la escala estuvo conformada por tres factores que explicaron un total del $52.24 \%$ de la varianza. El primero de ellos, el de Satisfacción, explicó el $18.55 \%$ de la varianza, quedó 
compuesto por los ítems 1-6 $(\mathrm{k}=6)$; el segundo, Eficiencia, explicó el $16.99 \%$ de la varianza, lo conformaron los ítems 2-6 $(\mathrm{k}=5)$; y el tercero, Efectividad, explicó el $16.71 \%$ de la varianza, lo constituyeron los ítems 2,3 , 5-7 $(\mathrm{k}=5)$.

\section{Análisis factorial confirmatorio}

Se realizó el análisis factorial confirmatorio (ver Figura 1) utilizando el software de ecuaciones de modelos estructurales AMOS 25 e implementado en SPSS. En relación a los índices de bondad de ajuste del modelo, se usaron los índices RMSEA, TLI y CFI. Los índices de bondad de ajuste del modelo de Usabilidad fueron RMSEA $=0.048, \mathrm{TLI}=0.95, \mathrm{CFI}=0.96, \mathrm{PClose}=0.64$ y X2/gl 232.00/98.

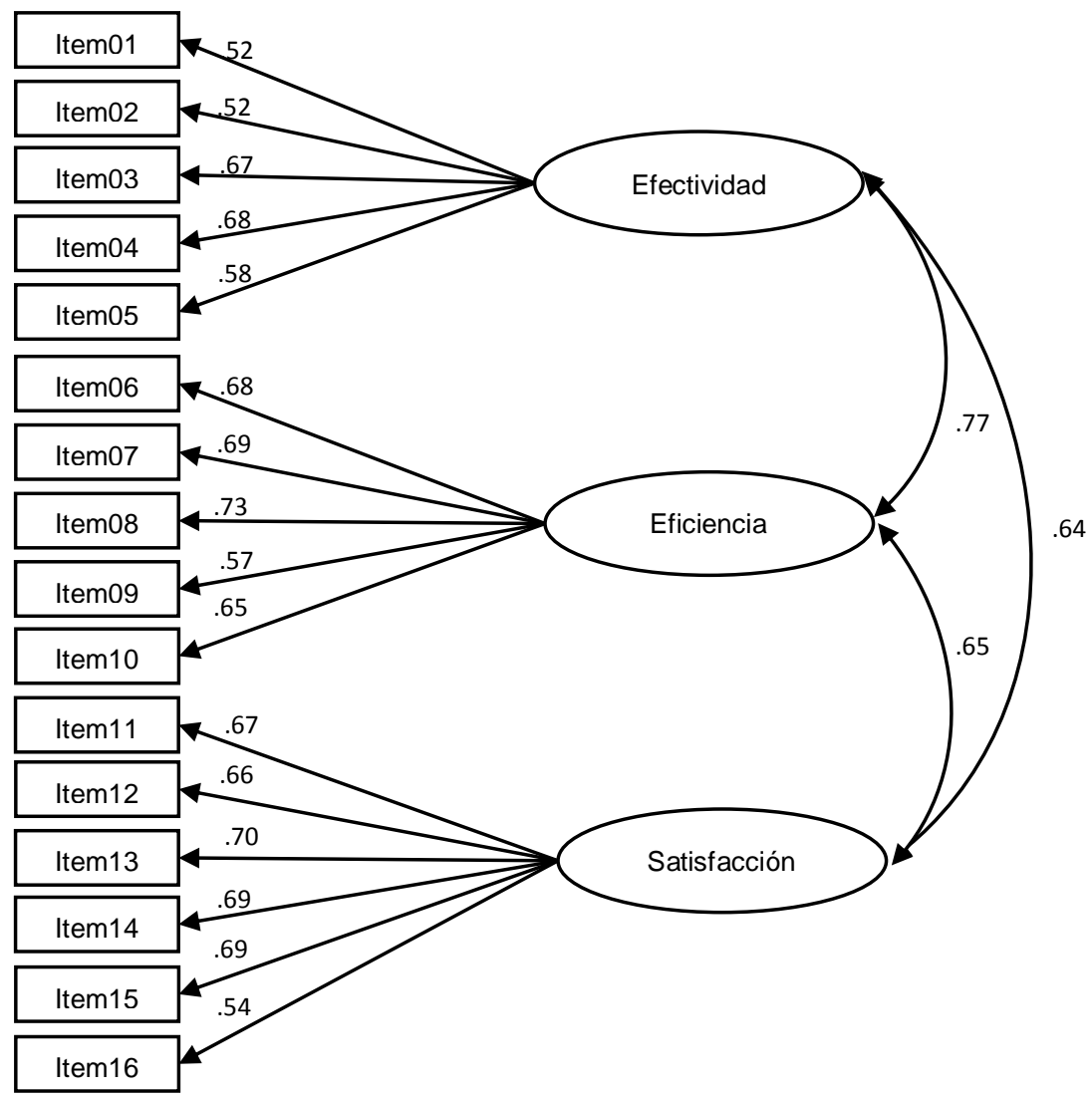

Fig. 1: Modelo de la escala de usabilidad. dimensiones: efectividad, eficiencia y satisfacción.

\section{Análisis de fiabilidad}

Como indicador de consistencia interna se consideró el alfa de Cronbach; el resultado para cada dimensión se presenta en la Tabla 2. La recolección de datos se realizó a través de la aplicación presencial por grupos y posteriormente se procesaron mediante el programa SPSS 23; el tamaño del efecto y la potencia estadística fueron calculados con $\mathrm{G}^{*}$ Power 3.1.9.2.

Tabla 2: Consistencia interna de la escala de usabilidad

\begin{tabular}{|l|c|c|}
\hline $\begin{array}{c}\text { Aspecto de la } \\
\text { usabilidad }\end{array}$ & $\begin{array}{c}\text { Ítems de la } \\
\text { escala }\end{array}$ & $\begin{array}{c}\text { Alfa de } \\
\text { Cronbach }\end{array}$ \\
\hline Efectividad & 5 & .77 \\
\hline Eficiencia & 5 & .83 \\
\hline Satisfacción & 6 & .83 \\
\hline
\end{tabular}

\section{RESULTADOS}

En este apartado se presentan los resultados del análisis descriptivo de los datos obtenidos al aplicar la escala de usabilidad con el propósito de determinar el uso de plataformas de redes sociales con fines académicos reportada por estudiantes universitarios a través de sus componentes y mediante estadística inferencial determinar si hay diferencias de dichos componentes entre los universitarios de ambos centros educativos utilizando una prueba de comparación de medias. 
Los análisis descriptivos de la escala de usabilidad se presentan en la Figura 2; en ella se identifican las medias de los puntajes por las dimensiones estudiadas. La dimensión con la media más alta fue la eficiencia en alumnos ITSON de 23.74 ( $D E=4.16)$ y una media de $22.95(D E=4.28)$ en alumnos ITSSAT; por lo que en ambas instituciones consideran que el usar comunidades en actividades académicas les facilita y agiliza la realización de actividades de aprendizaje. En la dimensión de satisfacción que hace referencia a la experiencia agradable al usar la plataforma de redes sociales para actividades académicas se obtuvieron puntajes similares entre los alumnos de ITSON $(\mathrm{M}=22.76, D E=4.12)$ y alumnos ITSSAT $(\mathrm{M}=22.18, D E=$ 3.71). Los puntajes más bajos se encontraron en la dimension de efectividad para ambas poblaciones, por lo que esto puede evidenciar la utilidad de la plataforma de redes sociales para actividades académicas; no obstante, en esta dimensión es donde se encontró una diferencia mayor en los puntajes del atributo. Como se puede apreciar también, la mayor diferencia se encuentra entre las medias de la efectividad con una diferencia cercana a 1.5; esta se reduce a 0.8 en la Eficiencia y a 0.6 en la Satisfacción atribuida al uso de comunidades virtuales para actividades académicas. Para identificar diferencias en los atributos se realizó una prueba $T$ de student.

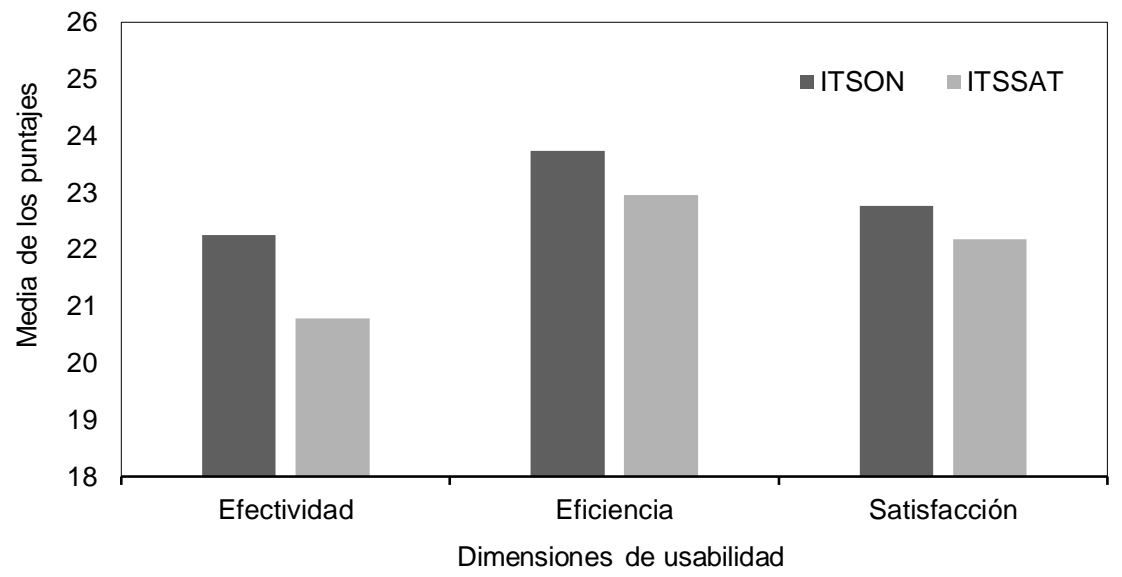

Fig. 2: Media de los puntajes por dimensión de la escala de usabilidad

\section{Análisis de medias}

Para identificar diferencias en los atributos se realizó una prueba t de Student. Se compararon las medias de los puntajes obtenidos en cada dimensión separado por institución educativa; el resumen del análisis se presenta en la Tabla 3. Para estos resultados se revisaron los supuestos de normalidad de los datos y se realizó la prueba de Levene para comprobar la igualdad de varianzas. Posteriormente, se aplicó una prueba t de comparación de medias. Adicionalmente, se reporta el tamaño del efecto (TE) y la potencia estadística para la cuantificación de la diferencia entre grupos.

Tabla 3: Resultados de la prueba t para comparar medias, potencia estadística y tamaño del efecto de las dimensiones.

\begin{tabular}{|l|c|c|c|c|c|c|c|}
\hline \multicolumn{1}{|c|}{ Dimensiones } & $M$ & $D E$ & $t$ & $g l$ & $p$ & $T E$ & $\begin{array}{c}\text { Potencia } \\
\text { estadística }\end{array}$ \\
\hline Efectividad & & & \\
\hline Sonora pública (ITSON) & 22.25 & 4.39 & 4.15 & 591 & .000 & 0.34 & 0.99 \\
\hline Veracruz pública (ITSSAT) & 20.79 & 4.09 & & & & \\
\hline Eficiencia & 23.74 & 4.17 & 2.26 & 591 & .024 & 0.18 & 0.73 \\
\hline Sonora pública (ITSON) & 23.95 & 4.29 & & & & & \\
\hline Veracruz pública (ITSSAT) & 22.9 & \\
\hline Satisfacción
\end{tabular}

Al hacer pruebas t para comparación de medias, se observó que las percepciones sobre las dimensiones de Usabilidad difieren significativamente $(p<0.05)$ en las dimensiones de Efectividad y Eficiencia, pero los valores de satisfacción no muestran diferencias significativas entre ambos grupos. Para interpretar apropiadamente, se calculó el tamaño del efecto que, de acuerdo a la clasificación de Cárdenas y Arancibia (2014), este es pequeño en la dimensión de efectividad $(0.344>.20)$; sin embargo, el tamaño del efecto en términos de eficiencia es cercano a .20, mientras que en satisfacción está por debajo del valor mínimo aceptado. Con el tamaño del efecto se calculó la potencia estadística que solo supera el nivel mínimo exigido (80\%) por lo que la probabilidad de cometer un error de tipo II es del $27 \%$ con los resultados de la dimensión de eficiencia y el $44.4 \%$ con el resultado de la prueba t para satisfacción. Por lo anterior, se consideró que la 
única diferencia significativa en los puntajes fue en la efectividad, ya que no hay diferencias en los atributos de Eficiencia y Satisfacción.

\section{DISCUSIÓN}

Aquí se analizan los resultados, sus implicaciones y el alcance de los mismos, considerando su relación con otros estudios similares. Los estudiantes mostraron actitudes positivas con respecto a las tres dimensiones de la usabilidad. Lo anterior concuerda con el estudio de Reyes-Garcés et al., (2018) quienes consideran que la personalización de Facebook permite adecuar e implementar "el uso de esta red social en el ámbito académico" (p. 73). Igualmente, hay coincidencia con los resultados de los estudios de Ansari (2020), Skendžić y Devčić (2017) y Tulinayo et al., (2018), en los cuales los estudiantes mostraron tendencias en usar las redes sociales con fines educativos principalmente para comunicarse con sus profesores, mejorar las experiencias educativas e intercambiar información con sus compañeros. De igual forma, Reyes-Garcés et al., (2018), observaron que sus estudiantes usaron un grupo de Facebook en un experimento, favoreciendo el aprendizaje colaborativo y la interacción en línea (Hussin et al., 2019). Por otra parte, en cuanto a las correlaciones del modelo se observó que a diferencia de la propuesta de lqbal y Warraich (2012), en el modelo propuesto en este estudio, las dimensiones de Efectividad y Eficiencia fueron las más altamente correlacionadas. En contraste con este estudio, Algahtani y Issa (2018) observaron que sus alumnos universitarios al empezar a usar las redes sociales para fines académicos, identificaron una serie de barreras de aceptación.

Referente a las dimensiones de eficiencia y satisfacción no difieren entre ambos grupos de alumnos. Lo anterior puede ser atribuido más a variables individuales entre las que se encuentran la calidad del aprendizaje en línea, el diseño y contenido del curso, las características del instructor y el estudiante, así como el apoyo técnico y administrativo (Puriwat y Tripopsakul, 2021) y a factores institucionales como el tamaño de la institución, infraestructura tecnológica o región donde se encuentra (Manca y Ranieri, 2016b). En cuanto a la diferencia encontrada en la efectividad entre ambos grupos, se considera que vale la pena explorar las prácticas de estudiantes y docentes que permiten considerar la utilidad de la plataforma para actividades académicas y abrir espacios de aprendizaje formales particularmente útiles en la educación a distancia.

\section{CONCLUSIONES}

De los resultados expuestos, y de su análisis y de discusión, se pueden obtener las siguientes conclusiones: En estudiantes de dos universidades mexicanas permite establecer estrategias adecuadas, seleccionar recursos adecuados para los alumnos y la aplicación de nuevos métodos que contribuyan a identificar otras variables asociadas a la implementación de tecnologías digitales en la educación: en este sentido, se deben determinar las funciones críticas para el desarrollo de actividades académicas de cada red social usada para la comunidad de aprendizaje, para determinar su pertinencia en términos de usabilidad con fines académicos, esto debido a que los alumnos de ambas universidades relacionan las redes sociales digitales como un espacio de esparcimiento y ocio, pero no con la educación, es así que prefieren separar ambas actividades.

\section{AGRADECIMIENTOS}

Agradecemos al Instituto Tecnológico de Sonora, por el financiamiento al proyecto a través del PROPAFI v2021; a los cuerpos académicos de Tecnología Educativa en la Sociedad del Conocimiento (CA - ITSON 27) e Innovaciones en Docencia, Investigación, y Extensión en Instituciones Educativas (CA-UV-101) de la UV, al Doctorado en Sistemas y Ambientes Educativos (PNPC - CONACYT), y a las autoridades de la Dirección de Ciencias Sociales y Humanidades, y al Departamento de Educación.

\section{REFERENCIAS}

Abbas, J., Aman, J., y otros dos autores, The Impact of Social Media on Learning Behavior for Sustainable Education: Evidence of Students from Selected Universities in Pakistan, https://doi.org/10.3390/su11061683, Sustainability, 11(6), $1-23$ (2019)

AIMX, 15e estudio sobre los hábitos de los usuarios de la Internet 2018, México (2019)

Algahtani, S., y Issa T., Barriers to the Adoption of Social Networking Sites in Saudi Arabia's higher education, https://doi.org/10.1080/0144929X.2018.1464600, Behaviour \& Information Technology, 37(10-11), 1072 - 1082 (2018)

Álvarez-Cruces, D. J., Otondo-Briceño, M., y Medina-Moreno, A., Evaluación de la Incorporación de un Foro Virtual por Redes Sociales entre Estudiantes de Odontología Chilenos, https://doi.org/10.18273/revsal.v51n2-2019004, Revista de la Universidad Industrial de Santander. Salud, 51(2), 117-128 (2019)

Ansari, J. A. N., y Khan, N. A., Exploring the Role of Social Media in Collaborative Learning the New Domain of Learning, https://doi.org/10.1186/s40561-020-00118-7, Smart Learning Environments, 7(9), 1-16 (2020)

Bond, M., y Bedenlier, S., Facilitating Student Engagement Through Educational Technology: Towards a Conceptual Framework, https://doi.org/10.5334/jime.528, Journal of Interactive Media in Education, 2019(1), 1-14 (2019) 
Cárdenas, M., y Arancibia, M. H., Potencia estadística y cálculo del tamaño del efecto en G* Power: complementos a las pruebas de significación estadística y su aplicación en psicología, https://doi.org/10.22199/S07187475.2014.0002.00006, Salud \& Sociedad, 5(2), 210-224 (2014)

Cattaneo, A., Motta, E., y Gurtner, J., Evaluating a Mobile and Online System for Apprentices' Learning Documentation in Vocational Education, https://doi.org/10.4018/IJMBL.2015070103, International Journal of Mobile and Blended Learning, 7(3), 40-58 (2015)

Chávez-Márquez, I. L., y Gutiérrez-Diez, M. C., Redes Sociales como Facilitadoras del Aprendizaje de Ciencias Exactas en la Educación Superior, Revista Apertura, ISSN: 2007-1094, 7(2), 49-61 (2015)

Garzón, M. A., Las Comunidades de Aprendizaje en las Organizaciones, Revista Visión de Futuro, ISSN: 1668-8708, 24(1) (2020)

Gómez-Hurtado, I., García-Prieto, F. J., y Delgado-García, M., Uso de la Red Social Facebook como Herramienta de Aprendizaje en Estudiantes Universitarios: Estudio Integrado sobre Percepciones, https://dx.doi.org/10.4151/07189729vol.57-iss.1-art.645, Perspectiva Educacional, 57(1), 99-119. (2018)

Guillén-López, O. B., Uso de Redes Sociales por Estudiantes de Pregrado de una Facultad de Medicina en Lima, Perú, https://doi.org/10.20453/rmh.v30i2.3550, Revista Médica Herediana, 30(2), 94-99 (2019)

Harrati, N., Bouchrika, I., y otros dos autores, Exploring user Satisfaction for E-learning Systems via Usage-based metrics and System Usability Scale Analysis, https://doi.org/10.1016/j.chb.2016.03.051, Computers in Human Behavior, 61(1), 463-471 (2016)

Hussin, W. N. T. W., Harun, J., y Shukor, N. A., Online Interaction in Social Learning Environment Towards Critical Thinking Skill: A Framework. Journal of Technology and Science Education, https://doi.org/10.3926/jotse.544, 9(1), 4-12 (2019)

Iqbal, M., y Warraich, N. F., Usability Evaluation of an Academic Library Website: A Case of the University of the Punjab, https://doi.org/10.47657/201213777, Pakistan Journal of Information Management and Libraries, 13, 32-42 (2012)

ISO 9241 - 11, Ergonomics of human - system interaction - Part 11: Usability: Definitions y concepts, 29, España, (2018)

ITSON, Cuarto Informe general de Actividades 2018-2019, Cd. Obregón, Sonora, México (2019)

ITSSAT, Reporte de oficialización de educación superior, SEP, México (2019)

Junco, R., Student Class Standing, Facebook Use, and Academic Performance,

https://doi.org/10.1016/j.appdev.2014.11.001, Journal of Applied Developmental Psychology, 36, 18-29 (2015)

Llorens, F., y Capdeferro, N., Posibilidades de la Plataforma Facebook para el Aprendizaje Colaborativo en Línea, RUSC. Universities and Knowledge Society Journal, E-ISSN: 1698-580X, 8(2), 31-45 (2011)

Manca, S., y Ranieri, M., Facebook and the Others. Potentials and Obstacles of Social Media for Teaching in Higher Education, https://doi.org/10.1016/j.compedu.2016.01.012, Computers \& Education, 95(1), 216-230 (2016a)

Manca, S., y Ranieri, M., "Yes for Sharing, no for Teaching!": Social Media in Academic Practices, https://doi.org/10.1016/j.iheduc.2015.12.004,The Internet and Higher Education, 29, 63-74 (2016b)

OCDE, Higher Education in Mexico: Labour Market Relevance and Outcomes of Higher Education in Four US States, OECD Publishing, Paris (2019)

Parsazadeh, N., Ali, R., Rezaei, M., y Tehrani, S. Z., The Construction and Validation of a Usability Evaluation Survey for Mobile Learning Environments, https://doi.org/10.1016/j.stueduc.2018.06.002, Studies in Educational Evaluation, 58, 97111, (2018)

Puriwat, W., y Tripopsakul, S., The Impact of e-Learning Quality on Student Satisfaction and Continuance Usage Intentions during COVID-19, https://doi.org/10.18178/ijiet.2021.11.8.1536, International Journal of Information and Education Technology, 11(8), 368-374 (2021)

Reyes-Garcés, E., Fernández-Peña, F., y otros dos autores, Aplicación Sigma y Grupos Facebook: Evaluación de la Usabilidad y Aceptación Tecnológica por Estudiantes de Ingeniería de Sistemas de la Universidad Técnica de Ambato en Ecuador, https://dx.doi.org/10.4067/S0718-50062018000500065, Formación Universitaria, 11(5), 65-74 (2018)

Rodríguez, K., Pérez, J. M. y Torres, G., Implementación de un Entorno Virtual como Herramienta Didáctica para Fortalecer el Proceso Enseñanza Aprendizaje, EDUMECENTRO, ISSN: 2077-2874, 10(4), 54-71 (2018)

Skendžić, A., y Devčić, K., The use of Social Networks for Educational Purposes - Case Study: Polytechnic Nikola Tesla in Gospic, https://dx.doi.org/10.18421/TEM63-24, TEM Journal, 6(3), 607-612 (2017)

Tulinayo, F. P., Ssentume, P., y Najjuma, R., Digital Technologies in Resource Constrained Higher Institutions of Learning: a Study on Students' Acceptance and Usability, https://doi.org/10.1186/s41239-018-0117-y, International Journal of Educational Technology in Higher Education, 15(36) (2018)

Zachos, G., Paraskevopoulou-Kollia, E. A., y Anagnostopoulos, I., Social Media Use in Higher Education: A Review, https://doi.org/10.3390/educsci8040194, Education Sciences, 8(4), 1-13 (2018) 